\title{
Assessment of Upper-Airway Configuration in Obstructive Sleep Apnea Syndrome With Computed Tomography Imaging During Müller Maneuver
}

\author{
Jie-Feng Huang MD, Gong-Ping Chen MD, Bi-Ying Wang MD, Han-Sheng Xie MD, \\ Jian-Ming Zhao MD, Li-Hua Wu MD, Li-Da Chen MD, and Qi-Chang Lin MD
}

BACKGROUND: The purpose of this observational study was to investigate the relationship between upper-airway configuration assessed by $\mathrm{CT}$ imaging during the Müller maneuver state and the severity of obstructive sleep apnea syndrome (OSAS). METHODS: A total of 358 snoring subjects who underwent standard polysomnography and upper-airway configuration by using CT imaging were enrolled. According to the apnea-hypopnea index (AHI), subjects were classified into 4 groups: snoring group (simple snoring), AHI $<5$; mild OSAS, $5 \leq$ AHI $<15$; moderate OSAS, $15 \leq \mathrm{AHI}<30$; and severe OSAS, AHI $\geq 30$. We also divided the upper airway into 3 parts, named the nasopharynx, oropharynx, and hypopharynx, from the CT scan and evaluated the minimal cross-sectional area (mCSA) and the shape of each airway level and calculated upper-airway length and distance from mandibular plane to hyoid bone (MPH). RESULTS: Multivariate logistic stepwise regression analysis identified body mass index (BMI), mCSA of nasopharynx, upper-airway length, and MPH as risk factors for the severity of OSAS. When subdivided for BMI and sex, upper-airway length was a risk factor for OSAS in non-obese (BMI $\left.<27 \mathrm{~kg} / \mathrm{m}^{2}\right)$ and male subjects, and MPH was a risk factor only in obese $\left(B M I \geq 27 \mathrm{~kg} / \mathrm{m}^{2}\right)$ subjects. Meanwhile, mCSA of nasopharynx was significantly associated with the severity of OSAS independent of BMI. CONCLUSIONS: Subjects with severe OSAS have more significant abnormalities of the upper airway. Obesity, mCSA of nasopharynx, upper-airway length, and MPH may contribute to the severity of OSAS. Obesity and sex should be taken into account when evaluating the abnormalities of upper-airway anatomy in snorers and patients with OSAS. Key words: obstructive sleep apnea syndrome; upper-airway configuration; CT; BMI; sex. [Respir Care 2016;61(12):1651-1658. () 2016 Daedalus Enterprises]

\section{Introduction}

Obstructive sleep apnea syndrome (OSAS) is an increasingly prevalent disease that is characterized by repet-

\footnotetext{
Drs Huang, Chen, Wang, Xie, Zhao, Wu, and Lin are affiliated with the Fujian Provincial Sleep-Disordered Breathing Clinic Center, Laboratory of Respiratory Disease of the Fujian Medical University, Department of Respiratory Medicine, First Affiliated Hospital of Fujian Medical University, Taijiang District, Fuzhou, Fujian Province, China. Dr Li-Da Chen is affiliated with the Department of Respiratory Medicine, Zhangzhou Affiliated Hospital of Fujian Medical University, Xiangcheng, Zhangzhou, Fujian Province, China.

This work was supported by Natural Science Foundation Grant 2014J01414 from Fujian Province, China. The authors have disclosed no conflicts of interest.
}

itive episodes of partial or complete upper-airway obstruction. Snoring has always been considered as one of the cardinal symptoms of OSAS, and snorers have a high risk of developing OSAS $^{1}$ and may even be in the early stage of OSAS.

It has been suggested that anatomical abnormalities of the upper airway, including upper-airway collapsibility, length and size, alterations in craniofacial structure, and enlarge-

\footnotetext{
Drs Huang, Chen, and Wang contributed equally to this work.

Correspondence: Qi-Chang Lin MD, NO 20, Chazhong Road, Taijiang District, Fuzhou, Fujian Province 350005, China. E-mail: chang4e@126.com.
}

DOI: $10.4187 /$ respcare.04669 
ment of surrounding soft tissue structures (ie, tongue and lateral pharyngeal walls), play an important role in the development of OSAS, ${ }^{2-7}$ and many authors have examined the shape and minimal cross sectional area (mCSA) at different levels of the upper airway in the transverse field, since the recurrent collapse of the upper airway during sleep is one of the most important pathogeneses in OSAS. ${ }^{3,6-9}$ Moreover, researchers also have examined the influence of body mass index (BMI) and sex on the anatomy of the upper airway. ${ }^{3,10,11}$ It has been suggested that non-obese patients with OSAS may have a higher incidence of upper-airway abnormalities. $^{3,11}$ Mayer et $\mathrm{al}^{3}$ found that in subjects with lower BMI, upper-airway abnormalities explain a major part of the variance in apnea-hypopnea index (AHI). However, Cosentini et al ${ }^{12}$ reported that obese subjects with OSAS had a very small minimal cross-sectional area with magnetic resonance imaging during wakefulness. In addition, results from previous studies have shown that the upper-airway length was significantly longer in men, which could partially explain the predisposition of men to OSAS compared with women, ${ }^{10,13}$ and it was also found that an inferiorly positioned hyoid, evaluated by distance from mandibular plane to hyoid bone (MPH), was commonly associated with OSAS. ${ }^{4,6}$ These controversial results suggest that different anatomical or physiological mechanisms may play the major role in the propensity to apnea in apnea patients of different BMI or sex.

Although most studies have been carried out by using CT to determine upper-airway configuration, most of them used a small sample size and did not investigate the effects of upper-airway length and MPH on the severity of OSAS simultaneously. Because one cannot expect a single anatomical abnormality to explain all of the variance in sleep apnea, upper-airway length and MPH, which have been reported to play an important role in the pathophysiology of OSAS, $3,5,7,14$ should also be taken into account when evaluating the relationship of upper-airway configuration and the severity of OSAS. Moreover, CT scan with the Müller maneuver state, which involves maximal inspiration against a closed nose and mouth and can well identify sites of upper-airway obstruction and reveals OSAS severity, ${ }^{15,16}$ has great advantages in detecting sites of upperairway obstruction.

In the present study, we evaluate the upper airway by CT scan with the Müller maneuver using the largest reported sample to date (358 subjects). We aim to investigate whether the severity of OSAS was associated with the structural changes of the upper airway induced by the Müller maneuver, even after stratification by BMI and sex of the subjects.

\section{QUICK LOOK}

\section{Current knowledge}

Anatomical abnormalities of the upper airway play an important role in the development of OSAS. Recurrent collapse of the upper airway during sleep induced by upper-airway abnormalities, including narrowing of the pharyngeal lumen and changing of pharyngeal shape, is one of the most important pathogeneses in OSAS, and BMI and sex have an effect on the anatomy of the upper airway.

\section{What this paper contributes to our knowledge}

Upper-airway abnormalities, including narrowing of nasopharynx, upper-airway length, and the distance from the mandibular plane to the hyoid bone, are likely to play an important physiopathogenic role in OSAS. Men have more upper-airway abnormalities, and obesity has a great effect on upper-airway length and the distance from the mandibular plane to the hyoid bone but not the area of the upper airway.

\section{Methods}

\section{Subjects}

Consecutive subjects who were referred to our sleep laboratory with a chief complaint of snoring from February 2013 to February 2015 were included to our study. Exclusion criteria were as follows: patients with asthma, COPD, cerebrovascular disease, symptomatic ischemic heart disease, congestive heart failure, chronic renal failure, hypothyroidism, and rheumatologic diseases according to self-reported medical histories. We also excluded patients with nasal, oral, pharyngeal, or mandibular diseases. Patients who had been previously diagnosed with or treated for OSA were also excluded. The final sample of this study comprised 358 subjects who met the inclusion criteria. Each subject had undergone both upper-airway CT scanning and a polysomnographic analysis (described below). This research was approved by institutional review board in the First Affiliated Hospital of Fujian Medical University.

\section{Medical History and Anthropometric Measurements}

Age, a detailed questionnaire on sleep symptoms, Epworth sleepiness scale, and history of alcohol consumption and smoking were recorded for all subjects. Body weight and height were measured without shoes and lightly clothed in the morning, and BMI was calculated as $\mathrm{kg} / \mathrm{m}^{2}$. Waist circumference was measured in the middle between the 
12th rib and the iliac crest, and neck circumference was measured at the level of the laryngeal prominence by a measuring tape.

\section{Polysomnography}

Polysomnography (P Series Sleep System, Compumedics, Melbourne, Australia) was performed overnight between 10:00 pm and 6:00 am, and the following parameters were recorded simultaneously: electroencephalography, electrooculography, electromyography, air flow by nasal and oral thermistors, respiratory effort by thoracic and abdominal impedance belts, arterial oxyhemoglobin saturation by pulse oximetry, snoring by tracheal microphone, and changing of the body position during sleep by sensor. According to the criteria of American Academy of Sleep Medicine published in 2012,17 the polysomnography recordings were manually scored for sleep stages by a physician. Apnea was defined as decrements in air flow of $\geq 90 \%$ from baseline for $\geq 10 \mathrm{~s}$. Hypopnea was defined as $\mathrm{a} \geq 30 \%$ decrease in flow, lasting $\geq 10 \mathrm{~s}$, accompanied by a $\geq 4 \%$ oxyhemoglobin saturation (or followed by an arousal when OSAS was diagnosed by overnight polysomnography). The AHI was calculated as the number of apneas and hypopneas/h of polysomnographically recorded sleep time. The oxygen desaturation index was defined as the number of dips in $\mathrm{S}_{\mathrm{pO}_{2}}$ of $\geq 4 \% / \mathrm{h}$ of polysomnographically recorded sleep time. Other polysomnographic parameters also included lowest $\mathrm{O}_{2}$ saturation, mean nocturnal oxygen saturation (average $\mathrm{S}_{\mathrm{pO}_{2}}$ ), and the percentage of sleep time with $\mathrm{S}_{\mathrm{pO}_{2}}<90 \%$. Subjects were divided into 4 groups with respect to OSAS severity based on AHI: snoring group (simple snoring), AHI $<5$; mild OSAS, $5 \leq$ AHI $<15$; moderate OSAS, $15 \leq$ AHI $<30$; and severe OSAS, AHI $\geq 30$. OSAS severity was defined as early OSAS when AHI was $<15$ events/h, and advanced OSAS was defined as when it was $\geq 15$ events/h. ${ }^{18}$

\section{CT Evaluation}

CT scan was used to measure anteroposterior and transverse diameters at the level of the nasopharynx (upper limit, cranial base; lower limit, tip of uvula), oropharynx (upper limit, tip of uvula; lower limit, tip of the epiglottis), and hypopharynx (upper limit, at the level of vallecula; lower limit, level of the cricoid cartilage) in the Müller maneuver state in all subjects. Slices in the axial plane, extending from the skull base to the hypopharynx, below the level of the cricoid cartilage, were collected in 5-mm intervals, with a total scan time of $10 \mathrm{~s}$. All of the subjects remained awake in the supine position with the Frankfort plane perpendicular to the floor accompanied. A lateral scout view was first taken to determine and standardize the level of the scans during quiet tidal breathing (Fig. 1A).

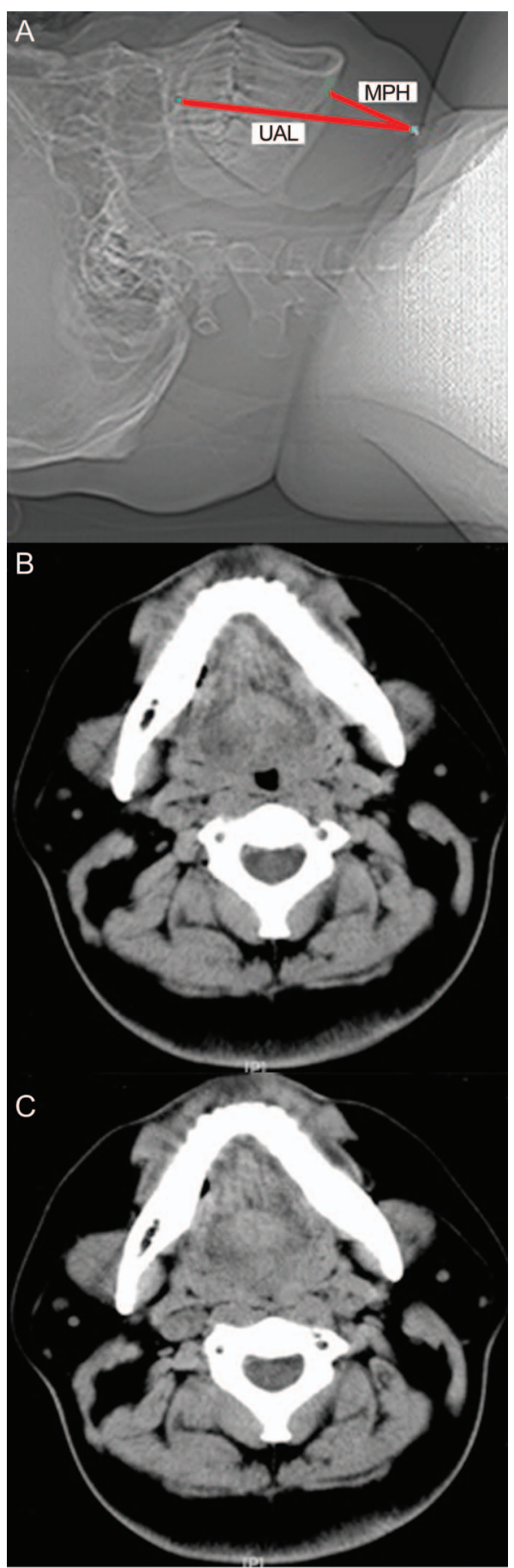

Fig. 1. Computed tomography using a lateral scout view (A) was first used to determine and standardize the level of the scans during quiet tidal breathing, extending from the skull base to the hypopharynx, below the level of the cricoid cartilage. Upper airway length (UAL) and the distance from the mandibular plane to the hyoid bone (MPH) were obtained from the lateral scout view. $\mathrm{B}$ : Cross-sectional image of a subject at the level of the nasopharynx in the Müller maneuver state. C: Complete obstruction of the airway at the level of the nasopharynx in the Müller maneuver state. 
UpPer-Airway CONFIgURATION IN OSAS By CT IMAging

Table 1. Anthropometric Characteristics and Polysomnography Parameters in All Subjects According to Obstructive Sleep Apnea Syndrome Status

\begin{tabular}{|c|c|c|c|c|c|}
\hline & Snoring Group & Mild OSAS & Moderate OSAS & Severe OSAS & $P$ \\
\hline Subjects, $n$ & 37 & 50 & 71 & 200 & \\
\hline \multicolumn{6}{|l|}{ Sex, $n(\%)$} \\
\hline Male & $51(56.8)$ & $36(72.0)$ & $65(91.5)$ & $181(90.5)$ & $<.001$ \\
\hline Female & $16(43.2)$ & $14(28.0)$ & $6(8.5)$ & $55(9.5)$ & \\
\hline Age, mean \pm SD y & $44.49 \pm 14.47$ & $46.94 \pm 10.54$ & $45.39 \pm 10.77$ & $45.52 \pm 11.94$ & .80 \\
\hline Current smoking, $n(\%)$ & $10(27.0)$ & $17(34.0)$ & $26(37.1)$ & $73(37.2)$ & .67 \\
\hline Alcohol consumption, $n(\%)$ & $1(2.7)$ & $4(8.0)$ & $7(10.0)$ & $42(21.5)$ & $<.001$ \\
\hline $\mathrm{BMI}$, mean $\pm \mathrm{SD} \mathrm{kg} / \mathrm{m}^{2}$ & $23.82 \pm 3.20$ & $25.30 \pm 2.85$ & $25.68 \pm 2.35$ & $28.03 \pm 5.83$ & $<.001$ \\
\hline Neck circumference, mean $\pm \mathrm{SD} \mathrm{cm}$ & $36.07 \pm 3.56$ & $37.67 \pm 3.88$ & $38.50 \pm 2.95$ & $40.09 \pm 3.12$ & $<.001$ \\
\hline Waist circumference, mean $\pm \mathrm{SD} \mathrm{cm}$ & $86.33 \pm 9.34$ & $90.36 \pm 8.66$ & $93.14 \pm 7.58$ & $98.11 \pm 10.45$ & $<.001$ \\
\hline AHI, median (IQR) events/h & $2.70(0.80-4.15)$ & $10.30(7.95-12.10)$ & $21.40(19.10-24.50)$ & $55.95(44.63-66.70)$ & $<.001$ \\
\hline ODI, median (IQR) events/h & $1.50(0.65-2.60)$ & $6.65(3.78-8.33)$ & $15.25(9.10-20.50)$ & $48.90(32.80-64.35)$ & $<.001$ \\
\hline T90\%, median (IQR) \% & $0.00(0.00,0.06)$ & $0.29(0.03,1.40)$ & $1.50(0.26,4.09)$ & $14.18(3.68,27.99)$ & $<.001$ \\
\hline $\mathrm{LaSO}_{2}$, median (IQR) \% & $90.00(86.00-91.00)$ & $85.00(80.00-88.00)$ & $80.00(76.00-84.00)$ & $67.00(56.00-78.00)$ & $<.001$ \\
\hline Average $\mathrm{S}_{\mathrm{pO}_{2}}$, median (IQR) $\%$ & $97.00(95.00-97.00)$ & $95.00(94.00-96.00)$ & $95.00(93.00-96.00)$ & $91.00(87.00-94.00)$ & $<.001$ \\
\hline ESS score, mean \pm SD & $7.08 \pm 4.34$ & $8.44 \pm 5.25$ & $7.45 \pm 5.62$ & $10.11 \pm 5.26$ & $<.001$ \\
\hline $\begin{array}{l}\mathrm{OSAS}=\text { obstructive sleep apnea syndrome } \\
\mathrm{BMI}=\text { body mass index } \\
\mathrm{AHI}=\text { apnea-hypopnea index } \\
\mathrm{IQR}=\text { interquartile range } \\
\mathrm{ODI}=\text { oxygen desaturation index } \\
\mathrm{T} 90 \% \text { = percentage of total sleep time spent with } \\
\mathrm{LaSO} \mathrm{S}_{2}=\text { lowest } \mathrm{O}_{2} \text { saturation } \\
\mathrm{ESS}=\text { Epworth sleepiness scale }\end{array}$ & $<90 \%$ & & & & \\
\hline
\end{tabular}

The mCSA within the nasopharynx, oropharynx, and hypopharynx regions (mCSA-nasopharynx, mCSA-oropharynx, and mCSA-hypopharynx) was obtained through measuring anteroposterior and transverse, and the shape at each mCSA level was expressed as the ratio of anteroposterior to transverse (anteroposterior/transverse nasopharynx, anteroposterior/transverse oropharynx, and anteroposterior/transverse hypopharynx) in the Müller maneuver state (Fig. 1, B and C). Upper-airway length was defined as the vertical distance from the hard palate to the hyoid in the mid-sagittal plane. We also calculated the MPH. Upper-airway length and MPH were obtained from the lateral scout view during quiet respiration within the first $5 \mathrm{~s}$ (Fig. 1A). We did not recognize any problematic images (eg, not in the neutral anatomical position) while reviewing the CT scan. Every subject was taught how to perform the Müller maneuver several times until they could perform the standard maneuver to avoid bias. All of the measurements were done manually by one clinician, who was blinded to the polysomnography data.

\section{Statistical Analysis}

All statistical analysis was performed by using SPSS 17.0 for Windows (SPSS, Chicago, Illinois). Before analysis, all variables were examined for normal distribution. Normally distributed, skewed, and categorical data are expressed as mean $\pm \mathrm{SD}$, median (interquartile range), and $n(\%)$, respec- tively. Then normally distributed continuous variables were analyzed by using one-way analysis of variance for multiplegroup comparison. Skewed data were compared by using Kruskal-Wallis $\mathrm{H}(\mathrm{K})$. All categorical variables were analyzed by the chi-square test or Fisher exact test. In view of the influence of BMI and sex on the anatomy of the upper airway, the correlation between AHI and clinical, CT data was evaluated in the overall population and in the different groups of BMI (using the cut-off point of $<27$ and $\geq 27 \mathrm{~kg} / \mathrm{m}^{23}$ ) and sex by Spearman's correlation test. Subjects were divided into early OSAS and advanced OSAS based on different treatment modalities, and stepwise logistic regression analyses were performed with the severity of OSAS as the dependent variable in the overall population and in the different groups of BMI and sex. Differences were considered significant when $P$ was $<.05$.

\section{Results}

A total of 358 subjects were evaluated in this study. Seventy-two percent of them were male, with the mean age of all subjects $45.6 \pm 11.8 \mathrm{y}$ and the mean BMI $26.75 \pm 4.95 \mathrm{~kg} / \mathrm{m}^{2}$. Based on the severity of OSAS, all of the subjects were divided into early OSAS ( $n=87,24.3 \%)$ and advanced OSAS $(n=271,75.7 \%)$.

Anthropometric characteristics and polysomnography parameters of subjects are summarized in Table 1. There were no significant differences among the 4 groups with 
UpPer-Airway CONFIgURATION IN OSAS By CT IMAging

Table 2. Upper-Airway Measurements in All Subjects According to the Presence of Obstructive Sleep Apnea Syndrome and Its Severity

\begin{tabular}{|c|c|c|c|c|c|}
\hline & Snoring Group & Mild OSAS & Moderate OSAS & Severe OSAS & $P$ \\
\hline $\mathrm{T}$-NP, median (IQR) cm & $1.55(1.23-2.00)$ & $1.41(1.16-1.72)$ & $1.45(0.98-1.77)$ & $0.98(0.66-1.45)$ & $<.001$ \\
\hline AP-NP, median (IQR) cm & $0.85(0.74-1.03)$ & $0.74(0.56-0.95)$ & $0.70(0.56-0.96)$ & $0.66(0.47-0.89)$ & $<.001$ \\
\hline $\mathrm{T}-\mathrm{OP}$, median (IQR) $\mathrm{cm}$ & $2.44(1.85-3.08)$ & $2.39(1.99-2.89)$ & $2.15(1.71-2.76)$ & $1.83(1.36-2.43)$ & $<.001$ \\
\hline AP-OP, median (IQR) cm & $1.22(0.83-1.55)$ & $1.28(1.00-1.60)$ & $1.27(1.03-1.50)$ & $1.22(0.94-1.59)$ & .96 \\
\hline T-HP, median (IQR) cm & $0.90(0.40-1.17)$ & $1.08(0.43-1.26)$ & $0.70(0.23-1.22)$ & $0.75(0.23-1.17)$ & .14 \\
\hline AP-HP, median (IQR) cm & $1.50(0.92-1.90)$ & $1.59(0.51-1.94)$ & $1.31(0.52-2.01)$ & $1.23(0.28-1.91)$ & .21 \\
\hline mCSA-NP, median (IQR) $\mathrm{cm}^{2}$ & $1.25(0.93-1.98)$ & $1.13(0.64-1.79)$ & $0.94(0.62-1.49)$ & $0.68(0.35-1.05)$ & $<.001$ \\
\hline mCSA-OP, median (IQR) $\mathrm{cm}^{2}$ & $2.93(1.64-4.36)$ & $3.12(2.31-4.14)$ & $2.70(2.00-3.76)$ & $2.25(1.44-3.36)$ & .01 \\
\hline mCSA-HP, median (IQR) $\mathrm{cm}^{2}$ & $1.10(0.37-1.94)$ & $1.71(0.21-2.39)$ & $0.90(0.13-2.39)$ & $1.06(0.07-2.04)$ & .19 \\
\hline AP/T-NP, median (IQR) & $0.54(0.45-0.73)$ & $0.50(0.37-0.75)$ & $0.56(0.41-0.70)$ & $0.64(0.47-0.94)$ & .01 \\
\hline AP/T-OP, median (IQR) & $0.38(0.49-0.63)$ & $0.52(0.42-0.67)$ & $0.54(0.43-0.77)$ & $0.64(0.48-0.93)$ & $<.001$ \\
\hline AP/T-HP, median (IQR) & $1.65(1.24-2.12)$ & $1.44(1.33-1.85)$ & $1.73(1.32-2.00)$ & $1.46(1.08-1.93)$ & .16 \\
\hline $\mathrm{UAL}$, mean $\pm \mathrm{SD} \mathrm{cm}$ & $6.71 \pm 1.28$ & $6.90 \pm 0.77$ & $7.16 \pm 0.67$ & $7.86 \pm 0.99$ & $<.001$ \\
\hline $\mathrm{MPH}$, mean $\pm \mathrm{SD} \mathrm{cm}$ & $1.70 \pm 1.04$ & $1.57 \pm 0.67$ & $1.71 \pm 0.65$ & $2.44 \pm 0.82$ & $<.001$ \\
\hline
\end{tabular}

$\mathrm{IQR}=$ interquartile range

$\mathrm{T}-\mathrm{NP}=$ transverse diameter at the mCSA level of nasopharynx

$\mathrm{T}-\mathrm{OP}=$ transverse diameter at the mCSA level of oropharynx

$\mathrm{T}-\mathrm{HP}=$ transverse diameter at the mCSA level of hypopharynx

AP-NP $=$ anteroposterior diameter at the mCSA level of nasopharynx

AP-OP $=$ anteroposterior diameter at the mCSA level of oropharynx

$\mathrm{AP}-\mathrm{HP}=$ anteroposterior diameter at the mCSA level of hypopharynx

mCSA-NP $=$ minimal cross sectional area within the nasopharynx region

$\mathrm{mCSA}-\mathrm{OP}=$ minimal cross sectional area within the oropharynx region

mCSA-HP $=$ minimal cross sectional area within the hypopharynx region

$\mathrm{AP} / \mathrm{T}=$ anteroposterior/transverse axis ratio

$\mathrm{UAL}=$ upper-airway length

$\mathrm{MPH}=$ distance from the mandibular plane to the hyoid bone

respect to age and current smoking. Polysomnographic parameters, including lowest $\mathrm{O}_{2}$ saturation and average $\mathrm{S}_{\mathrm{pO}_{2}}$, decreased significantly with an increase in OSAS severity, whereas alcohol consumption, BMI, neck circumference, waist circumference, AHI, oxygen desaturation index, and percentage of sleep time with $\mathrm{S}_{\mathrm{pO}_{2}}<90 \%$ increased with OSAS severity.

Table 2 presents upper-airway measurements in the Müller maneuver state. No significant differences were observed in terms of anteroposterior oropharynx, transverse hypopharynx, anteroposterior hypopharynx, and mCSAhypopharynx among groups. Among the subjects with severe OSAS, transverse nasopharynx, anteroposterior nasopharynx, and transverse oropharynx were $0.98,0.66$, and $1.83 \mathrm{~cm}$, decreased significantly when compared with the snoring group $(P<.001,<.001$, and $<.001$, respectively). The mCSA-nasopharynx and mCSA-oropharynx among subjects with severe OSAS were smaller when compared with the snoring group $\left(0.68 \mathrm{~cm}^{2}\right.$ vs $1.25 \mathrm{~cm}^{2}$ $[P<.001]$ and $2.25 \mathrm{~cm}^{2}$ vs $2.93 \mathrm{~cm}^{2}[P<.001]$, respectively). Considering the anteroposterior/transverse ratio at the level of nasopharynx and oropharynx, the severe OSAS group tended to present a more spherical shape at the level of nasopharynx and of oropharynx. In addition, a strong positive association was observed between OSAS severity and the indices of upper-airway length and MPH (both $P<.001)$.

Table 3 summarizes the associations between upperairway measurements and polysomnographic parameters in the overall population and in the different groups of BMI and sex. There were significant correlations between $\mathrm{AHI}$ and mCSA and the shape of the upper airway, including mCSA-nasopharynx, mCSA-oropharynx, mCSAhypopharynx $(\mathrm{r}=-0.366,-0.181$, and $-0.140 ; P<.001$, $<.001$, and .01, respectively). We also observed positive correlations between $\mathrm{AHI}$ and the upper-airway length and MPH ( $\mathrm{r}=0.521$ and 0.481 ; both $P<.001)$. Additionally, Spearman's correlation also revealed a significant association between AHI and sex and BMI $(r=0.242$ and 0.484 ; both $P<.001)$. Afterwards, in the BMI class $<27 \mathrm{~kg} / \mathrm{m}^{2}$, mCSA-nasopharynx, mCSA-oropharynx, upper-airway length, MPH, sex, and BMI were significantly correlated with AHI. In the BMI class $\geq 27 \mathrm{~kg} / \mathrm{m}^{2}$, mCSA-nasopharynx, upper-airway length, MPH, sex, and BMI were significantly correlated with AHI. In the subgroup of males, mCSA-nasopharynx, mCSA-oropharynx, mCSAhypopharynx, upper-airway length, MPH, and BMI were significantly correlated with AHI, whereas only age, BMI, and mCSA-nasopharynx were significantly correlated with AHI in the group of females. 
Table 3. Spearman's Rank Correlation Coefficients Between Polysomnography Parameters, Anthropometric Characteristics, and Upper-Airway Measurements

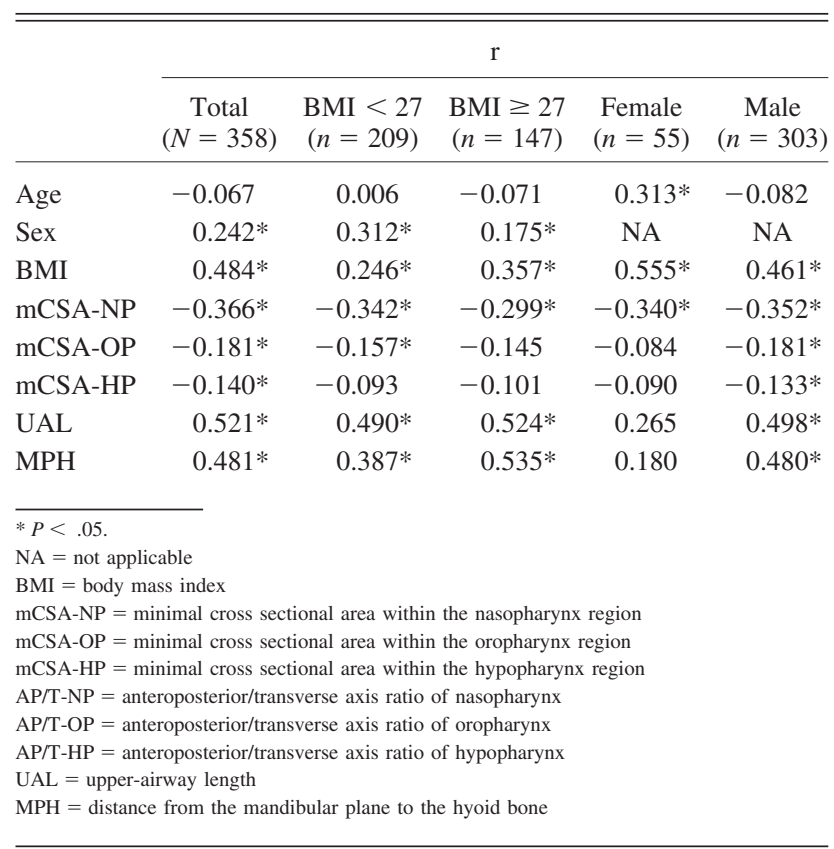

For the overall population, multivariate stepwise logistic regression analyses showed the following factors as determinants of the severity of OSAS: BMI, mCSA-nasopharynx, upper-airway length, and MPH. In the group with a BMI $<27 \mathrm{~kg} / \mathrm{m}^{2}$, sex, mCSA-nasopharynx, and upperairway length were associated with the severity of OSAS, whereas mCSA-nasopharynx and MPH emerged in the group with a BMI $\geq 27 \mathrm{~kg} / \mathrm{m}^{2}$. In the subgroup of males, anteroposterior/transverse oropharynx and upper-airway length were the independent variables for the severity of OSAS, whereas only age and BMI were significantly correlated with AHI in the group of females (Table 4).

\section{Discussion}

The present study provides evidence that there were significant correlations between mCSA and the shape of the upper airway and the severity of OSAS in the Müller maneuver state. In the logistic regression model, besides BMI, mCSA-nasopharynx, upper-airway length, and MPH were found to be significant risk factors for OSAS. Meanwhile, when subjects were classified according to BMI and sex, multivariate logistic stepwise regression analysis revealed that upper-airway length was a significant risk factor only in non-obese male subjects with OSAS, and MPH was a significant factor in obese subjects. Another important new finding was that subjects were commonly characterized by upper-airway narrowing at the level of nasopharynx independent of BMI.
Table 4. Multivariate Stepwise Logistic Regression Analysis Models for Different Groups According to Severity of Obstructive Sleep Apnea Syndrome

\begin{tabular}{|c|c|c|c|}
\hline Variable & $P$ & Odds Ratio & $95 \% \mathrm{CI}$ \\
\hline \multicolumn{4}{|l|}{ Overall } \\
\hline BMI & $<.001$ & 1.246 & $1.114-1.394$ \\
\hline mCSA-NP & .025 & 0.641 & $0.435-0.945$ \\
\hline UAL & .002 & 1.644 & $1.198-2.256$ \\
\hline MPH & .01 & 1.640 & $1.109-2.424$ \\
\hline \multicolumn{4}{|c|}{$\mathrm{BMI}<27 \mathrm{~kg} / \mathrm{m}^{2}$} \\
\hline Sex & .047 & 2.636 & $1.014-6.856$ \\
\hline mCSA-NP & $<.001$ & 0.628 & $0.411-0.958$ \\
\hline UAL & .002 & 2.019 & $1.283-3.178$ \\
\hline \multicolumn{4}{|c|}{$\mathrm{BMI} \geq 27 \mathrm{~kg} / \mathrm{m}^{2}$} \\
\hline mCSA-NP & .048 & 0.481 & $0.233-0.994$ \\
\hline $\mathrm{MPH}$ & .001 & 4.882 & $1.898-12.560$ \\
\hline \multicolumn{4}{|l|}{ Female } \\
\hline Age & .02 & 1.079 & $1.014-1.148$ \\
\hline BMI & .004 & 1.375 & $1.108-1.706$ \\
\hline \multicolumn{4}{|l|}{ Male } \\
\hline AP/T-OP & .008 & 6.148 & $1.607-23.517$ \\
\hline UAL & $<.001$ & 2.428 & $1.593-3.701$ \\
\hline \multicolumn{4}{|c|}{$\begin{array}{l}\text { BMI = body mass index } \\
\text { mCSA-NP = minimal cross sectional area within the nasopharynx } \\
\text { UAL = upper-airway length } \\
\text { MPH = distance from the mandibular plane to the hyoid bone } \\
\text { AP/T-OP = anteroposterior/transverse axis ratio of oropharynx }\end{array}$} \\
\hline
\end{tabular}

The mechanisms responsible for upper-airway collapse and apnea were incompletely understood in snorers and patients with OSAS. Previously, it had been reported that upper-airway collapse induced by upper-airway abnormalities, including narrowing of the pharyngeal lumen and changing of pharyngeal shape, leads to snoring and apnea during sleep $3,8,19,20$; however, few researchers had focused on mCSA and the shape of the upper airway in the Müller maneuver state. It is believed that the the Müller maneuver, a method to simulate upper-airway collapsibility under intraluminal pressure during sleep, has been validated in sleep apnea, and collapse of the pharynx induced by the Müller maneuver is highly related to severity of OSAS. ${ }^{15,18,21}$ In the present study, mCSA of nasopharynx, oropharynx, and hypopharynx all had a negative correlation with OSAS severity in the Müller maneuver state, and mCSA of nasopharynx was an independent variable for OSAS following multiple logistic regression analyses in both obese and non-obese subjects. Our results were similar to those of smaller studies using CT to identify change in the upper airway. ${ }^{8,20}$ However, some studies did not find any significant differences in the upper-airway area between OSAS and control subjects, ${ }^{3,7}$ even those grouped by BMI. ${ }^{3}$ In addition, we also found in our study that a more spherical shape of the oropharynx plays a more important role in the development of OSAS in male subjects 
with an odds ratio of 6.148 (95\% CI 1.607-23.517). Mayer et $\mathrm{al}^{3}$ showed that the shapes of the oropharynx and hypopharynx were more spherical in the higher BMI group, which could not be found in this study. The influence of the breathing cycle and state may partly account for the inconsistent conclusion.

In this study, we also have demonstrated that upperairway length and MPH were significantly correlated with AHI, as expected. Some researchers have elucidated the importance of upper-airway length in potentially explaining pharyngeal collapse. $5,7,14$ Sutherland et al ${ }^{14}$ investigated the effect of weight loss on upper-airway length in obese men and suggested that reduced upper-airway length was associated with a greater improvement in OSAS. The correlation between upper-airway length change and its collapsibility could be explained based on Bernoulli's law, which states that an inviscid liquid flowing through a tube is susceptible to an increase in velocity and decrease in pressure. ${ }^{22}$ According to the rules of buckling of cylindrical shells, ${ }^{23}$ upper-airway length would increase, and the upper airway would be increasingly prone to collapse because it lacks rigid bony structures. Therefore, it seems that upper-airway length is a possible mechanism contributing to increased upper-airway collapsibility in patients with OSAS. Our data also showed that the upper-airway length might be a dominant contributing factor for obstructive sleep apnea in men or non-obese patients. These findings were in accordance with previous studies in subjects with OSAS ${ }^{5,7}$ but were discussed here for the first time, to our knowledge, in non-obese subjects with OSAS.

Another finding in this study was that MPH was an independent variable for significant OSAS with an odds ratio of 1.640 (95\% CI 1.109-2.424) in all subjects and 4.882 (95\% CI 1.898-12.560) in obese subjects. The hyoid bone, which serves as anchorage for the tongue muscles, affects the patency of the hypopharyngeal airway, and inferiorly positioned hyoid bone in OSAS has been well documented. 4,6,20 In one study, it was found that the vertical distance of the hyoid position decreased after weight loss, suggesting that this phenomenon results from a lessening of downward pressure due to a reduction in excess pharyngeal tissues. ${ }^{14}$ In agreement with the previous reports, our study also indicated a more inferior hyoid bone position likely to play an important physiopathogenic role in OSAS, especially in obese patients.

Previous studies have also reported that cephalometric features and the upper-airway abnormalities were more important risk factors for OSAS in non-obese compared with obese subjects. ${ }^{3,24,25}$ However, in the study of Tangugsorn et al, ${ }^{26}$ subjects with OSAS displayed aberrations of cervico-craniofacial and upper-airway soft tissue morphology irrespective of the BMI. These conflicting results suggest that the influence of obesity on patient abnormalities remains to be established. The results of our study showed that abnormalities such as narrowing of the pharyngeal lumen were found in obese and non-obese subjects regardless of BMI, whereas upper-airway length was the major determinant in non-obese subjects and MPH in the obese group. It has been speculated that these abnormalities possibly imply a genetic predisposition to OSAS in such patients. ${ }^{3}$ Furthermore, we also noted a sex difference in the upper-airway abnormalities and found that men had more predisposing factors than did women, including a more spherical shape of the oropharynx and more inferiorly positioned hyoid bones, which might partly explain why being male is a major risk factor for OSAS. These findings in our study suggest that the etiology of OSAS is multifactorial, and obesity, sex, and subtle abnormalities in upper-airway configuration are important factors in its pathogenesis.

The present study clearly has several limitations. First, the CT scan was conducted on awake subjects, whereas OSAS is a dynamic phenomenon occurring as patients are asleep. However, it is not practical to perform CT imaging on sleeping patients. Thus, we evaluated upper airways in the Müller maneuver state, which was a method to simulate upper-airway collapsibility during sleep. ${ }^{21}$ Second, from an ethical viewpoint, CT in OSAS diagnosis puts patients at risk of radiation exposure compared with other clinically used diagnostic methods, such as nasopharyngoscopy and magnetic resonance imaging. However, CT scanning had advantages, including providing multiplelevel, rapid-sequence scans, sensitivity to both soft tissue and bony landmarks, the ability to combine it with polysomnography, and the noninvasive nature of the technique. Third, we only selected a few of the potentially important variables that may determine the impact of airway configuration on sleep apnea severity instead of examining all of the parameters of airway configuration. Other measurements that may be important include soft-tissue thickness around the airway, tongue length, and others. We chose measurements that were believed to be simple and intuitively correlated to the severity of sleep apnea to avoid problems with biases related to repeated measures.

\section{Conclusions}

Upper-airway abnormalities, including narrowing of the nasopharynx, upper airway-length, and MPH are more likely to play a more important physiopathogenic role for OSAS in the overall population following multiple logistic regression analyses. Our study also suggests that men have more upper-airway abnormalities and that obesity has a great effect on upper-airway length and the distance from mandibular plane to the hyoid bone but not the area of the upper airway. The precise mechanism underlying the association with obesity, sex, and subtle abnormalities in upper-airway configuration requires further clarification. 


\section{UpPeR-Airway CONFIGURATION IN OSAS By CT IMAGING}

\section{REFERENCES}

1. Puvanendran K, Goh KL. From snoring to sleep apnea in a Singapore population. Sleep Res Online 1999;2(1):11-14.

2. Maltais F, Carrier G, Cormier Y, Series F. Cephalometric measurements in snorers, non-snorers, and patients with sleep apnoea. Thorax 1991;46(6):419-423.

3. Mayer P, Pépin JL, Bettega G, Veale D, Ferretti G, Deschaux C, Lévy P. Relationship between body mass index, age and upper airway measurements in snorers and sleep apnoea patients. Eur Respir J 1996;9(9):1801-1809.

4. Hui DS, Ko FW, Chu AS, Fok JP, Chan MC, Li TS, et al. Cephalometric assessment of craniofacial morphology in Chinese patients with obstructive sleep apnoea. Respir Med 2003;97(6):640-646.

5. Segal Y, Malhotra A, Pillar G. Upper airway length may be associated with the severity of obstructive sleep apnea syndrome. Sleep Breath 2008;12(4):311-316.

6. Thapa A, Jayan B, Nehra K, Agarwal SS, Patrikar S, Bhattacharya D. Pharyngeal airway analysis in obese and non-obese patients with obstructive sleep apnea syndrome. Med J Armed Forces India 2015; 71(Suppl 2):S369-S375.

7. Kim EJ, Choi JH, Kim YS, Kim TH, Lee SH, Lee HM, et al. Upper airway changes in severe obstructive sleep apnea: upper airway length and volumetric analyses using 3D MDCT. Acta Otolaryngol 2011; 131(5):527-532.

8. Caballero P, Alvarez-Sala R, García-Río F, Prados C, Hernán MA, Villamor J, Alvarez-Sala JL. CT in the evaluation of the upper airway in healthy subjects and in patients with obstructive sleep apnea syndrome. Chest 1998;113(1):111-116.

9. Wu Z, Chen W, Khoo MC, Davidson Ward SL, Nayak KS. Evaluation of upper airway collapsibility using real-time MRI. J Magn Reson Imaging. 2016;44(1):158-167

10. Ronen O, Malhotra A, Pillar G. Influence of gender and age on upper-airway length during development. Pediatrics 2007;120(4): e1028-e1034.

11. Yu X, Fujimoto K, Urushibata K, Matsuzawa Y, Kubo K. Cephalometric analysis in obese and nonobese patients with obstructive sleep apnea syndrome. Chest 2003;124(1):212-218.

12. Cosentini T, Le Donne R, Mancini D, Colavita N. Magnetic resonance imaging of the upper airway in obstructive sleep apnea. Radiol Med. 2004;108(4):404-416.

13. Malhotra A, Huang Y, Fogel RB, Pillar G, Edwards JK, Kikinis R, et al. The male predisposition to pharyngeal collapse: importance of airway length. Am J Respir Crit Care Med 2002;166(10):1388-1395.
14. Sutherland K, Lee RW, Phillips CL, Dungan G, Yee BJ, Magnussen JS, et al. Effect of weight loss on upper airway size and facial fat in men with obstructive sleep apnoea. Thorax 2011;66(9):797-803.

15. Kim HY, Bok KH, Dhong HJ, Chung SK. The correlation between pharyngeal narrowing and the severity of sleep-disordered breathing. Otolaryngol Head Neck Surg 2008;138(3):289-293.

16. Ko MT, Su CY. Computer-assisted quantitative evaluation of obstructive sleep apnea using digitalized endoscopic imaging with Muller maneuver. Laryngoscope 2008;118(5):909-914.

17. Grigg-Damberger MM. The AASM Scoring Manual four years later. J Clin Sleep Med 2012;8(3):323-332.

18. Wu MJ, Ho CY, Tsai HH, Huang HM, Lee PL, Tan CT. Retropalatal Muller grade is associated with the severity of obstructive sleep apnea in non-obese Asian patients. Retropalatal Muller grade and OSA in non-obese. Sleep Breath 2011;15(4):799-807.

19. Liu SY, Huon LK, Lo MT, Chang YC, Capasso R, Chen YJ, et al Static craniofacial measurements and dynamic airway collapse patterns associated with severe obstructive sleep apnea: a sleep MRI study. Clin Otolaryngol. 2015. doi: 10.1111/coa.12598.

20. Lam B, Ooi CG, Peh WC, Lauder I, Tsang KW, Lam WK, Ip MS Computed tomographic evaluation of the role of craniofacial and upper airway morphology in obstructive sleep apnea in Chinese. Respir Med 2004;98(4):301-307.

21. Borowiecki B, Pollak CP, Weitzman ED, Rakoff S, Imperato J. Fibro-optic study of pharyngeal airway during sleep in patients with hypersomnia obstructive sleep-apnea syndrome. Laryngoscope 1978; 88(8 Pt 1):1310-1313.

22. Fajdiga I. Snoring imaging: could Bernoulli explain it all? Chest 2005;128(2):896-901.

23. Ekstrom RE. Buckling of cylindrical shells under combined torsion and hydrostatic pressure. Exp Mech 1963;3(8):192-197.

24. Sakakibara H, Tong M, Matsushita K, Hirata M, Konishi Y, Suetsugu S. Cephalometric abnormalities in non-obese and obese patients with obstructive sleep apnoea. Eur Respir J 1999;13(2): 403-410.

25. Ito D, Akashiba T, Yamamoto H, Kosaka N, Horie T. Craniofacial abnormalities in Japanese patients with severe obstructive sleep apnoea syndrome. Respirology 2001;6(2):157-161.

26. Tangugsorn V, Krogstad O, Espeland L, Lyberg T. Obstructive sleep apnoea: multiple comparisons of cephalometric variables of obese and non-obese patients. J Craniomaxillofac Surg 2000;28(4):204212. 